\title{
Type 1 Diabetes Mellitus Patient Presented with Insulin Edema only after Intensive Lifestyle Changes: A Case Report and Brief Review of the Literature
}

\author{
Aida Al Jabri'1,2,3, Zahra Buali' \\ ${ }^{1}$ Department of Pediatrics, King Abdulaziz Hospital, Ministry of the National Guard-Health Affairs, Al-Ahsa, KSA \\ ${ }^{2}$ King Abdullah International Medical Research Center, Al-Ahsa, KSA \\ ${ }^{3}$ King Saud Bin Abdulaziz University for Health Sciences, Al-Ahsa, KSA \\ Email: dr_aida_s@hotmail.com
}

How to cite this paper: Al Jabri, A. and Buali, Z. (2021) Type 1 Diabetes Mellitus Patient Presented with Insulin Edema only after Intensive Lifestyle Changes: A Case Report and Brief Review of the Literature. Case Reports in Clinical Medicine, 10, 381-386.

https://doi.org/10.4236/crcm.2021.1011047

Received: October 21, 2021

Accepted: November 8, 2021

Published: November 11, 2021

Copyright $\odot 2021$ by author(s) and Scientific Research Publishing Inc. This work is licensed under the Creative Commons Attribution International License (CC BY 4.0).

http://creativecommons.org/licenses/by/4.0/

\begin{abstract}
Insulin edema is considered a rare type of generalized edema that occurs after initiation or intensification of insulin treatment in patients with diabetes mellitus (DM). In this case report, we present a clinically significant edema that developed following a dramatic improvement in glycemic control after intensive improvement in lifestyle in a 13-year-old girl, with type $1 \mathrm{DM}$ confirmed by positive anti-glutamic acid decarboxylase (anti-GAD) antibodies. She had poor glycemic control during 2 years of insulin therapy, and following lifestyle improvements (with no changes to her medications types or doses), she developed generalized body edema, and facial puffiness that resolved spontaneously, without any intervention.
\end{abstract}

\section{Keywords}

Insulin Edema, Type 1 Diabetes, Type 2 Diabetes

\section{Introduction}

Generalized edema is a rare complication of insulin therapy, which usually occurs in newly diagnosed type 1 diabetic patients and after the initiation of insulin therapy. Also it can occur in patients with type 2 diabetes after the intensification of insulin therapy. Generalized edema must be differentiated from other causes of edema such as cardiac or renal disease which are the most common causes of body edema, and must initially be ruled out [1].

Here we report a 13-year-old, girl, who was diagnosed with type $1 \mathrm{DM}$ in 
2016. Her DM was uncontrolled for 2 years, and she had a history of multiple admissions to the pediatric intensive care unit, due to diabetes ketoacidosis (DKA). She recently presented with generalized body edema including the face, of no apparent cause, after sudden improvement in her glycemic control with only lifestyle modifications, and without any change in insulin types or doses.

\section{Case Presentation}

A 13-year-old Saudi girl diagnosed with type 1 diabetes in 2016, had positive anti-GAD antibodies, and in 2018, had uncontrolled blood glucose with a high hemoglobin A1c (HgA1c) level of more than 15\%. Her lifestyle was unsatisfactory, including the consumption of many junk foods with lots of carbs, fried foods, and soft drinks and a lack of any physical exercise. Despite her good insulin dose of 1.5 units/kg/day and good compliance to her medication, she still had uncontrolled blood glucose and was frequently admitted to the pediatric intensive care unit (PICU) as a case of diabetic ketoacidosis (DKA) with a total of 5 admissions since her diagnosis, and her HgAlc level reached $16.9 \%$ on one occasion. We decided to provide her with intensive psychological support and gave her frequent clinical appointments with the pediatrics endocrinology clinic and a diabetes educator. She decided to improve her quality of life by changing her lifestyle and preventing any diabetic complications, therefore, she changed her types of food that she consumed, eliminated most of carbs, became physically active and, walking around for an hour per day.

In September 2020 she was admitted to the PICU due to frequent hypoglycemic attacks, with no history of fatigue or decreased energy, no symptoms of cough, shortness of breath, or chest pain, and no history of new medication use. A physical examination determined that she had pitting edema on her lower limbs, lower back, and a puffy face. She had warm skin with no tingling or burning sensations at the site of edema and no pain, aching, or numbness. Other systemic exams showed she was within normal, a bodyweight of $58 \mathrm{~kg}$, and had a BMI of $23 \mathrm{~kg} / \mathrm{m}^{2}$, with no abnormalities in her chest, heart or abdomen. A normal thyroid exam and showed no lipohypertrophy at the injection site. Investigations were conducted to rule out any organic cause of the edema, included renal function test, liver function test, and cardiac exams with ECG. All were normal apart from her albumin being on the lower side with a level of $35 \mathrm{~g} / \mathrm{L}$. Other labs tests showed a high HgAlc level of 15.8\%. ACTH and cortisol tests were performed to rule out Addison's disease and the results were within normal ranges. The celiac profile showed negative antibodies. Thyroid function tests revealed a normal TSH, low FT4 level of $8.2 \mathrm{pmol} / \mathrm{L}$ and positive thyroid antibodies. Insulin doses were adjusted with the total daily dose decreasing from 1.5 to 1.3 and then 0.7 units/kg/day to control her blood glucose level, and her long-acting insulin dose was changed to another type of long-acting insulin (Tresiba) to minimize her hypoglycemic attacks. On clinical follow-up, she showed much improvement in her glycemic status, and her edema resolved without any medical interventions. Her last measured HA1c level was $7.8 \%$, 
levothyroxine was initiated with a dose of $50 \mathrm{mcg}$ daily, and her last FT4 level was $10.3 \mathrm{pmol} / \mathrm{L}$.

Her HgAlc level decreased from $15.8 \%$ to $9.1 \%$ and then decreased again in December 2020 to $7.9 \%$.

\section{Discussion}

Insulin edema is a known complication of the initiation or intensification of insulin therapy. It was first described in 1928 [2] and occurs in both type 1 and type 2 diabetes mellitus, especially in newly diagnosed cases of type 1 diabetes mellitus [1]. It is a benign transient condition, that is usually self-limiting and resolves spontaneously with a rare or no need to use any intervention, treatment, or diuretic therapy [3].

Although the manifestations can vary widely from mild peripheral edema to cardiac failure and massive serosal effusions and may occur in local areas, such as the periorbital, pretibial, and sacral regions, or as generalized edema, there was a young patient who is diagnosed as a newly diagnosed type 1 diabetes, when generalized edema and pericardial effusion developed after initiation of insulin therapy, the treatment was only supportive and all symptoms resolved within 20 days [3] [4]. As well, it can present at any age with the youngest age was reported was a 9 years old male who developed insulin edema few days after diagnosis of type 1 diabetes and was treated as a case of DKA [2].

There are no criteria or risk stratification systems for the diagnosis of insulin edema, and it is diagnosed by the exclusion of other common causes of body edema. However, aged being from 20 to 40 years, poor control diabetes either by having frequent hypoglycemia or following DKA treatment, newly diagnosed diabetes with higher doses of insulin therapy, poor glucose control, and low body mass index have been suggested as risk factors for insulin edema [5] [6].

The true incidence of insulin edema remains unknown but some reports have estimated the incidence to be in the range of $3 \%-3.5 \%$ [1].

There have been several presentations of insulin edema, William et al. reported a 15-year-old girl with a history of poorly controlled type 1 diabetes mellitus who presented with generalized anasarca which started gradually over her limbs till it extended to all of her body including her face and genitalia associated with a significant increase in her weight, her labs were within normal level apart of albumin which was in the lower normal, these symptoms were noticed after 1-week of compliance with insulin therapy [7]. Furthermore, another adult patient with latent autoimmune diabetes was reported to have insulin edema associated with neuropathy. She was a 35-year-old, diagnosed with diabetes mellitus and subsequently developed latent autoimmune diabetes. She was on an oral hypoglycaemic agent and her glucose level was high with an HbA1c of $13 \%$. She was admitted to the hospital under plastic surgery service when she presented with a painful discoloration of her right finger. During that admission, she developed DKA, after treatment of the DKA insulin was initiated and after 3 days 
she developed significant pitting edema up to her umbilicus associated with neuropathy that was diagnosed by nerve conduction studies. Other causes of edema were excluded, and because her edema was severe so, she was treated with furosemide and fluid restriction [6]. Another adult case was reported by Wong and Balakrishnan. The patient was a 40-year-old woman, newly diagnosed with type 1 diabetes mellitus who developed generalized edema just after diagnosis and treatment initiation [1]. Sugiyama et al. reported that a 50-year-old female patient who is known to have acute myelogenous leukemia (AML) post bone marrow transplantation and type 2 diabetes mellitus, was on multiple medications as prophylaxis and treatment from transplant rejection. Her blood glucose became poorly controlled so, intensive insulin therapy was given to her. After around 4 weeks, she was found to have insulin edema over her lower limbs [8]. The same presentation was found in a 57-year-old male patient with a 5-year history of type 2 diabetes, he was poorly controlled and his urinalysis showed positive ketones and protein. He developed bilateral lower limb edema which was induced by the rapid improvement of glycemic control with insulin therapy [9]. Despite that, insulin edema is a rare finding and usually, it is self-resolved, although it can be serious with significant complications as in this patient who was a 35-year-old woman diagnosed recently with type 1 diabetes who presented with polydipsia and polyuria for over 1 year, and at the last 2 months before presentation, she lost approximately $2 \mathrm{~kg}$ of weight. She presented with signs of severe DKA indicated by Kussmaul's breathing and low $\mathrm{pH}$ levels and was treated with insulin. On the 2nd day, her renal function started to deteriorate, she had high creatinine levels and then presented with generalized edema, serous effusions, and acute renal failure. This case was different due to the presence of more serious complications [10]. Pediatric patients also can have insulin edema. A case report of a 12-year-old boy newly diagnosed with type 1 diabetes, presented with edema of the lower extremities approximately one week after the initiation of insulin therapy. This case was considered a typical presentation of insulin edema that appeared soon after the initiation of insulin therapy in a patient newly diagnosed with type 1 DM [11]. Furthermore, B. Hirshberg et al. reported a 49-year-old female, who was diagnosed with diabetes mellitus and developed severe generalized edema following the initiation of insulin therapy. The edema was severe enough to be treated with diuretics but unfortunately, it was impossible to continue the treatment because of the severe side effects of this medication the patient experienced [12]. Even the patients who are on an insulin pump can develop insulin edema, Greco and his colleagues described the case of a 39-year-old woman with poorly controlled type 1 diabetes mellitus who developed generalized edema with severe weight gain a few days after the start of an insulin pump therapy [3].

\section{Conclusion}

Several cases have been reported about generalized edema in patients with di- 
abetes. However, most of the cases reported on patients with either newly diagnosed type 1 or type 2 diabetes mellitus that developed edema after the initiation or intensification of insulin treatment or even after sudden compliance with insulin therapy. There were no case reports about the development of insulin edema after only improvements in the patient's lifestyle and without any change in insulin dose.

\section{Conflicts of Interest}

The authors declare no conflicts of interest regarding the publication of this paper.

\section{References}

[1] Wong, M. and Balakrishnan, T. (2020) Anasarca in Newly Diagnosed Type 1 Diabetes: Review of the Pathophysiology of Insulin Edema. Cureus, 12, e7234. https://doi.org/10.7759/cureus.7234

[2] Aravamudhan, A., Gardner, C., Smith, C. and Senniappan, S. (2014) Insulin Oedema in a Child with Newly Diagnosed Diabetes Mellitus. European Journal of Pediatrics, 173, 685-687. https://doi.org/10.1007/s00431-013-2045-0

[3] Greco, D. (2015) Severe Weight Gain and Generalized Insulin Edema after the Starting of an Insulin Pump. Canadian Journal of Diabetes, 39, 21-23. https://doi.org/10.1016/j.jcjd.2014.07.001

[4] Moravej, H., Karamizadeh, Z., Nikfarjam, F. and Amoozgar, H. (2012) Pericardial Effusion in a Teenaged Type 1 Diabetic Patient after Insulin Therapy. Iranian Journal of Pediatrics, 22, 570-571.

[5] Amrapala, A. and Chowdhury, T.A. (2019) Severe Oedema in a Patient with Diabetes. Clinical Medicine, 19, 325. https://doi.org/10.7861/clinmedicine.19-4-325

[6] Tufton, N., Coppack, S. and Huda, B. (2015) An Unusual Cause of Generalized Insulin Edema and Truncal Neuropathy. Journal of Medical Cases, 6, 563-565.

https://doi.org/10.14740/jmc2345w

[7] Myers, W.T., Wintergerst, K.A., Kingery, S.E. and Foster, M.B. (2016) Case 2: Generalized Anasarca in 15-Year-Old Girl with Type 1 Diabetes Mellitus. Pediatrics in Review, 37, 81-82. https://doi.org/10.1542/pir.2015-0088

[8] Sugiyama, A., Takeuchi, S., Fukagawa, S., Moroi, Y., Yoshimoto, G., Miyamoto, T., et al. (2012) Case of Insulin Edema Occurring during Intensive Insulin Therapy after Bone Marrow Transplantation. The Journal of Dermatology, 39, 172-175. https://doi.org/10.1111/j.1346-8138.2011.01309.x

[9] Kan, Y., Anno, T., Kawasaki, F., Takai, M., Shigemoto, R., Kaneto, H., Kaku, K. and Okimoto, N. (2017) Bilateral Lower Limb Edema Induced by Rapid Improvement of Glycemic Control with Insulin Therapy in a Subject with Poorly Controlled Type 2 Diabetes. Acta Diabetologica, 54, 611-613. https://doi.org/10.1007/s00592-017-0997-7

[10] Laroche, S., Wucher, H., Nguyen, Y.-L., Timsit, J. and Larger, E. (2013) Case Report: Insulin Edema and Acute Renal Failure. Diabetes Care, 36, e65. https://doi.org/10.2337/dc12-2137

[11] Bulus, A.D., Andiran, N. and Köksal, A.O. (2016) Insulin Edema in Type 1 Diabetes Mellitus: Report of a Case and Brief Review of the Literature. Iranian Journal of Pediatrics, 26, e5077. https://doi.org/10.5812/ijp.5077 
[12] Hirshberg, B., Muszkat, M., Marom, T. and Muszkat, M. (2000) Natural Course of Insulin Edema. Journal of Endocrinological Investigation, 23, 187-188.

https://doi.org/10.1007/BF03343704 